\title{
American Thyroid Association Consensus Review and Statement Regarding the Anatomy, Terminology, and Rationale for Lateral Neck Dissection in Differentiated Thyroid Cancer
}

\author{
Brendan C. Stack, Jr. (Chair), Robert L. Ferris, ${ }^{2}$ David Goldenberg, ${ }^{3}$ Megan Haymart, ${ }^{4}$ \\ Ashok Shaha, ${ }^{5}$ Sheila Sheth, ${ }^{6}$ Julie Ann Sosa, ${ }^{7}$ and Ralph P. Tufano, ${ }^{6, *}$ \\ for the American Thyroid Association Surgical Affairs Committee
}

Background: Cervical lymph node metastases from differentiated thyroid cancer (DTC) are common. Thirty to eighty percent of patients with papillary thyroid cancer harbor lymph node metastases, with the central neck being the most common compartment involved. The goals of this study were to: (1) identify appropriate methods for determining metastatic DTC in the lateral neck and (2) address the extent of lymph node dissection for the lateral neck necessary to control nodal disease balanced against known risks of surgery.

Methods: A literature review followed by formulation of a consensus statement was performed.

Results: Four proposals regarding management of the lateral neck are made for consideration by organizations developing management guidelines for patients with thyroid nodules and DTC including the next iteration of management guidelines developed by the American Thyroid Association (ATA). Metastases to lateral neck nodes must be considered in the evaluation of the newly diagnosed thyroid cancer patient and for surveillance of the previously treated DTC patient.

Conclusions: Lateral neck lymph nodes are a significant consideration in the surgical management of patients with DTC. When current guidelines formulated by the ATA and by other international medical societies are followed, initial evaluation of the DTC patient with ultrasound (or other modalities when indicated) will help to identify lateral neck lymph nodes of concern. These findings should be addressed using fine-needle aspiration biopsy. A comprehensive neck dissection of at least nodal levels IIa, III, IV, and Vb should be performed when indicated to optimize disease control.

\section{Introduction}

CERVICAL (OR REgIONAL) lymph node metastases from differentiated thyroid cancer (DTC) are very common, particularly in patients with papillary thyroid cancer (PTC). Depending on the detection method employed, $30 \%-80 \%$ of patients with PTC harbor lymph node metastases, mostly in the central neck (1-4). The clinical significance of these metastases remains controversial. Although nodal disease increases the risk of recurrence, especially when lymph node metastases are macroscopic, the impact of microscopic lymph node metastases on recurrence and survival is less clear (510). Increases in the use and improvement in the resolution of a number of imaging modalities and the increasing sensitivity

The authors are members of the Subcommittee for Lateral Neck Dissection for Well-Differentiated Thyroid Cancer, of the American Thyroid Association (ATA) Surgical Affairs Committee, which assumes responsibility for the content of this article. Subcommittee members are alphabetically listed after the chair.

${ }^{*}$ Co-chair, ATA Surgical Affairs Committee.

${ }^{1}$ Department of Otolaryngology/Head and Neck Surgery, University of Arkansas for Medical Sciences, Little Rock, Arkansas.

${ }^{2}$ Department of Otolaryngology/Head and Neck Surgery, University of Pittsburgh, Pittsburgh, Pennsylvania.

${ }^{3}$ Division of Otolaryngology/Head and Neck Surgery, Department of Surgery, Penn State/Milton S. Hershey Medical Center, Hershey, Pennsylvania.

${ }^{4}$ Department of Medicine, University of Michigan, Ann Arbor, Michigan.

${ }^{5}$ Department of Surgery, Memorial Sloan-Kettering Cancer Center, New York, New York.

${ }^{6}$ Department of Otolaryngology/Head and Neck Surgery, Johns Hopkins University School of Medicine, Baltimore, Maryland.

${ }^{7}$ Divisions of Endocrine Surgery and Surgical Oncology, Department of Surgery, Yale University School of Medicine, New Haven, Connecticut. 
of thyroglobulin $(\mathrm{Tg})$ assays has made it possible to detect very small volume, persistent and/or recurrent disease, emphasizing the need to define the appropriate extent of surgery necessary to optimize regional control.

Optimizing the surgical approach is fundamental to appropriate initial management of DTC and involves balancing the risks and benefits of thyroidectomy and neck dissection $(11,12)$. Because DTC is more indolent than squamous cell carcinoma metastatic to the lymph nodes of the neck, it is not possible to universally extrapolate from one disease to the other. The primary goals of this interdisciplinary consensus review were to identify methods for determining when metastatic DTC is present in the lateral neck and to address the extent of lymph node dissection appropriate for control of the lateral neck supported by contemporary medical literature, balanced against the known risks of surgical therapy.

Regional lymph node spread from thyroid cancer can broadly be classified as central neck compartment and lateral neck compartment metastases. Lymph node metastases are most commonly found in the central neck compartment (level VI and level VII). The central neck dissection anatomy and terminology have been previously defined by the American Thyroid Association (ATA) Consensus Statement on the Terminology and Classification of Central Neck Dissection published in the November 2009 issue of Thyroid (13). Although less frequent, metastases to lymph nodes in the lateral neck, levels I-V may be associated with a worse prognosis (14). There is a clear association between central neck involvement and the likelihood of lateral neck disease, as an increase in the number of positive central neck lymph nodes is associated with a higher likelihood of lateral neck disease $(15,16)$.

The more extensive dissection required for lateral neck lymph node metastases is associated with cosmetic concerns, such as a longer incision, and the potential for nerve injury (accessory, marginal mandibular, sympathetic, phrenic, vagus, hypoglossal, cervical sensory branches, brachial plexus, and greater auricular nerves), hemorrhage, and chyle leak that are not associated with thyroidectomy alone with or without central neck dissection $(17,18)$. Complications from lateral neck dissections can be as high as $50 \%$, with a $3.6 \%$ incidence of chyle leak and an $11 \%$ or greater incidence of chronic neck pain and numbness (19). Some studies show that the addition of a lateral neck dissection to central neck compartment surgery (thyroidectomy or central neck dissection) doubles the risk of transient postoperative hypoparathyroidism $(19,20)$. Strict indications for lateral neck dissection for DTC are necessary to optimize a benefit profile expected to outweigh the risks of the procedure. Many philosophies for managing the lateral neck in DTC are presented in the literature, including elective nodal sampling, ultrasound (US) directed compartment resections, and super selective nodal dissection that is, levels III-IV, leading to some controversy regarding when and how to manage the lateral neck in DTC (21).

A subcommittee on Lateral Neck Dissection from the Surgical Affairs Committee of the ATA was organized and tasked with reviewing the existing literature regarding lateral neck dissection and DTC. Additionally, this subcommittee was tasked to review the salient anatomy and imaging as it related to the lateral neck and provide recommendations for consideration in the next iteration of the ATA management guide-

lines for patients with thyroid nodules and DTC and future guidelines on this topic from other sources (22). In the present report the authors, all of whom are members of this subcommittee, present a review of this topic and provide consensus opinions and recommendations.

\section{Review}

\section{Clinical factors in detection of lateral lymph node metastasis}

A number of important factors in the patient's history are relevant to the risk for and detection of lateral neck metastases in patients with DTC (Table 1). First, it is important to consider other risk factors, including but not limited to the patient's age, gender, tumor size, presence of lymphovascular invasion, extra-thyroidal extension, and presence of central compartment nodal disease. Younger patients and men appear to have a significantly higher risk of cervical lymph node metastasis than older patients or women (23). Recent identification of molecular alterations that predict more aggressive disease, such as the presence of the BRAF mutation (24), may be important markers to consider when assessing the risk for lateral neck metastasis. Consistent with the 2009 ATA guidelines (14), BRAF or other markers being positive alone are insufficient justification to do a prophylactic lateral neck dissection at the present time.

In the setting of primary or recurrent DTC, features such as palpable hard/firm lymph nodes should prompt a thorough and comprehensive assessment of the lateral neck by clinical and radiographic examination. Moreover, in the 2009 Revised ATA management guidelines for patients with thyroid nodules and DTC (14), hitherto referred to as ATA Thyroid cancer guidelines, recommendation 21 suggests that preoperative high resolution US examination of the contralateral thyroid lobe and cervical lymph nodes is the standard for all patients undergoing thyroid surgery for malignant cytology (14). Central neck nodes can be challenging to image in the

\section{Table 1. A Systematic Review of Literature as of September 2010 of Various Factors Affecting Lateral Neck Nodal Metastases in Well Differentiated Thyroid Cancer}

Parameter which affects presence of lateral nodal disease in DTC

References

Primary size

Age

Thyroid capsular invasion

Central neck disease

Type of neck dissection

Biomarkers

Recurrence in the lateral neck

Multifocality of primary disease

Follicular thyroid cancer

Hurthle cell cancer

AJCC stage

Distant metastases

Gender

Outcomes for lateral neck metastases Effect of neck dissection

AJCC, American Joint Commission on Cancer; DTC, differentiated thyroid cancer.
$(48,51,63)$

$(52,60)$

$(48,57)$

$(49,58)$

$(11,35,47,50,57,58,61)$

(53-56)

$(11,35,47,50,57,58,61)$

(59)

$(62,63)$

$(34,48,63)$

(9)

$(52,63)$

$(34,51)$

(61) 
Table 2. Ultrasound Guidelines for Cervical Node Level, Anatomic Boundary, and Optimal Transducer

\begin{tabular}{lll}
\hline Nodal level & \multicolumn{1}{c}{ Anatomic landmark } & Transducer \\
\hline I A & Midline above thyroid bed & Linear 8-12 MHz \\
I B right and left & Submandibular glands & Linear 8-12 MHz \\
II A right and left & Lateral to CCA upper neck & Linear 8-12 MHz \\
III right and left & Lateral to CCA midneck & Linear 8-12 MHz \\
IV right and left & Lateral to CCA lower neck & Linear 8-12 MHz \\
V A right and left & Lateral to sternocleidomastoid & Linear 8-12 MHz \\
VI right and left. Up, mid, low & Medial to CCA thyroid bed & Linear 8-12 MHz \\
V B Supraclavicular regions right and left & clavicle & Sector 4-6 MHz \\
\hline
\end{tabular}

CCA, common carotid artery.

preoperative setting due to the presence of an intact thyroid gland, but no such limitation exists for lateral neck imaging. US-guided biopsy of sonographically suspicious nodules and/or lymph nodes in the lateral neck should be performed whenever possible (Table 2) (14). Cystic lymph nodes should be approached with a high degree of suspicion as they are frequently positive cytologically or by $\mathrm{Tg}$ (25). On occasions, cytologic diagnosis is not possible due to scanty material obtained from fine-needle aspiration biopsy (FNAB), and the FNAB should be repeated. Thyroglobulin washings of FNAB aspirates may also be helpful in this situation and can be reliable even in the presence of circulating thyroglobulin antibodies (26). If the node or nodule is not amenable to biopsy or will not render a diagnosis by cytologic evaluation or $\mathrm{Tg}$ aspirate assay, watchful waiting for growth on serial USs may be prudent.

With a history of thyroid surgery for cancer and an elevated or rising serum Tg level, a thorough search for persistent and/ or recurrent/residual disease should include assessment of the lateral neck, initially using high resolution US examination. Nuclear medicine scanning may also be a part of the diagnostic evaluation of recurrence. Disease burden is roughly reflected in the $\mathrm{Tg}$ level in relation to the serum thyroid stimulating hormone (TSH) level; TSH provocation of $\mathrm{Tg}$ response can be elicited by direct TSH injection, or by thyroid hormone withdrawal and secondary TSH elevation. Positive Tg measurements measured in a state of a suppressed TSH are more concerning for recurrent/residual/bulky disease. Reliability of the $\mathrm{Tg}$ assay and the presence of interfering anti-thyroglobulin antibodies are important considerations when evaluating absolute Tg levels or trends.

\section{Definition of the lateral neck anatomy}

The stated goals of developing classifications for neck dissection are to uniformly define the clinical and surgical boundaries of each of the lymph node groups removed in a neck dissection and to develop a classification that correlates with the biology of cervical metastases thus meeting the compartmental excision standards following basic oncologic principles (27) (Fig. 1). This classification was used to describe the patterns of metastatic dissemination observed in more than 1000 squamous cell carcinoma patients who were treated with neck dissections from a surgeon's perspective (28). Lymph nodes in the lateral neck are grouped into levels I-V, corresponding with the submandibular and submental nodes (level I); upper, middle, and lower jugular nodes (levels II, III, IV respectively); and posterior triangle nodes (level V). Levels
VI and VII, commonly referred to as the central neck have already been recently well described and will not be dealt with further in this article (13). This system of nodal levels has been widely accepted, allowing data to be reported without ambiguity and among health care providers who perform oncologic neck surgery. Boundaries of these levels are:

- Level I: (Submandibular; submental) nodes are defined by the body of the mandible superiorly, stylohyoid muscle posteriorly, and the anterior belly of the digastric muscle on the contralateral side anteriorly. This level may be subdivided into a single midline level Ia, the submental triangle (bounded by the anterior bellies of

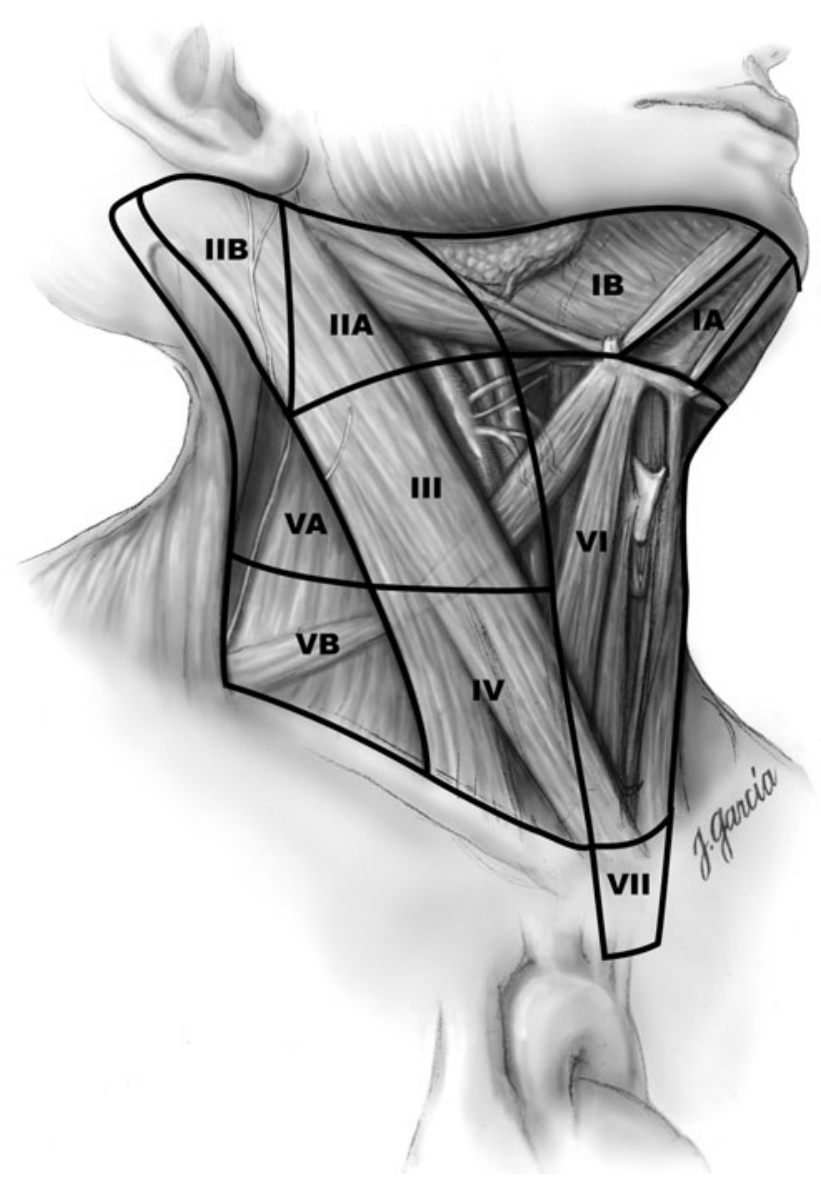

FIG. 1. Nodal levels with corresponding anatomic landmarks (used with permission from R. Udelsman, M.D.). 
the bilateral digastric muscles and the hyoid bone), and $\mathrm{Ib}$, containing the submandibular gland and its nodes.

- Level II: (Upper jugular) are located by the upper third of the jugular vein; it extends from the skull base to the inferior border of the hyoid bone. The anterior border of level II is the stylohyoid muscle, and the posterior border is the posterior border of the sternocleidomastoid muscle (SCM). The spinal accessory nerve, which travels obliquely across this area, is used as a landmark to subdivide this group into $\mathrm{Ilb}$, the portion above and behind the nerve, and IIa, the portion that lays anteroinferiorly. This level contains the jugulodigastric nodes.

- Level III: (Mid jugular) nodes are located between the hyoid above and a horizontal plane defined by the inferior border of the cricoid cartilage below. The sternohyoid muscle demarcates the anterior limit of level III, and the posterior border of the SCM is the posterior border of this level.

- Level IV: (Lower jugular) nodes are adjacent to the lower third of the jugular vein. These nodes are located between the inferior border of the cricoid cartilage and the clavicle, and like level III, the anterior boundary is the sternohyoid muscle, and the posterior boundary is the posterior border of the SCM. Thyroid cancer may cause prominent metastatic disease in this region. This level may contain many lymph vessels which connect to the thoracic duct on the left.

- Level V: (Posterior triangle) is bound anteriorly by the posterior border of the SCM and posteriorly by the anterior border of the trapezius muscle. This level extends from the convergence of the sternocleidomastoid and trapezius muscles superiorly to the clavicle inferiorly. This level is subdivided by a plane defined by the inferior border of the cricoid cartilage into level Va superiorly and level $\mathrm{Vb}$ inferiorly. Level $\mathrm{Va}$ contains the nodes surrounding the spinal accessory nerve, and level $\mathrm{Vb}$ contains the transverse cervical and supraclavicular nodes.

Subzones were established in levels I, II, and V in response to the increasing awareness that specific sublevels had lower or higher risk for nodal metastases (29): levels $\mathrm{Ia}, \mathrm{Ib}$, Ila, IIb, $\mathrm{Va}$, and $\mathrm{Vb}$. In 2008, further modifications were proposed, adopting radiographic landmarks and creating alternative borders to define levels I and II: the vertical plane defined by the posterior edge of the submandibular gland instead of, or in addition to, the clinically visible border of the stylohyoid muscle. It was further proposed that the medial aspect of the common carotid artery be an alternate landmark for separating the medial border of levels III and IV from the lateral border of level VI, which had traditionally been the lateral border of the sternohyoid muscle (30).

Imaging is an integral part of the assessment of thyroid cancer patients. The use of imaging in all of its forms has proliferated over the last three decades. Imaging findings often complement the physical examination, and an imagingbased classification provides the radiologist with guidelines for classifying the location of cervical nodes and communicating these findings to clinicians. Although the level numbering system is the same, some fine nuances separating nodal levels are introduced by the image-based system. Som et al. proposed the following computerized tomography (CT)based node level classifications (31):
- Level I: All nodes above the hyoid bone, below the mylohyoid muscle, and anterior to a transverse line drawn on each axial image through the posterior edge of the submandibular gland.

- Level II: Extends from the skull base at the lower level of the bony margin of the jugular fossa, to the level of the lower body of the hyoid bone. Level IIA nodes are level II nodes that surround the internal jugular vein (IJV). Level IIB nodes lie posterior to the spinal accessory cranial nerve $(\mathrm{CN})$ deep to the SCM and are separated from the IJV by a fat plane.

- Level III nodes lie between the level of the lower body of the hyoid bone and the level of the lower margin of the cricoid cartilage.

- Level IV nodes lie between the level of the lower margin of the cricoid cartilage arch and the level of the clavicle on each side, as seen on axial CT scan.

- Level V nodes extend from the skull base at the posterior border of the attachment of the SCM to the level of the clavicle. Level Va nodes lie between the skull base and the level of the lower margin of the cricoid cartilage arch, behind the posterior edge of the SCM. Level $\mathrm{Vb}$ nodes lie between the level of the lower margin of the cricoid cartilage and the level of the clavicle (31).

\section{Classification of neck dissections}

Consistency in description of lateral neck dissection is crucial to allow for a comparison of outcomes in those series reported in the literature. For example, it is insufficient to use qualifiers such as modified radical neck dissection without designating what levels were removed and what associated structures, if any, were sacrificed. Radical neck dissection is defined as removal of lymph nodes from levels I-V and resection of the IJV, the spinal accessory nerve (CN XI), and the SCM. This was originally described by George Crile in 1906, and it resulted in significant associated morbidity, and it is infrequently used today $(32,33)$. Modified radical dissection describes comprehensive removal of nodal basins (levels I-V) with preservation of one or more of the following nonlymphatic structures: CN XI, IJV, or SCM. A selective neck dissection refers to removal of less than all five nodal levels, and is directed by the patterns of lymphatic drainage from the primary tumor, while preserving CN XI, IJV, and SCM $(34,35)$. This is the most commonly used neck dissection in the management of lateral neck metastasis for thyroid cancer and should be reported with a designation of side and nodal levels and sublevels dissected (i.e., selective neck dissection of levels IIa, III, IV, and Vb).

\section{Lateral neck dissection}

The complications of lateral neck dissection are significant and must be factored into the equation of risks and benefits. The primary complication considered for lateral neck dissection is injury to the eleventh $\mathrm{CN}$. Although this injury is rarely caused by nerve transection, the acts of dissection and retraction might be sufficient to cause temporary or permanent weakness in up to $20 \%$ of patients (36). This risk is increased when levels IIb and Va are dissected. Patients experience debilitating shoulder droop and inability to raise the arm above the horizon. In some chronic cases, shoulder 
movement can be quite limited, painful, and disabling ("shoulder syndrome").

The most frequent permanent complication is numbness of the lateral neck and ear. This results from injury to the greater auricular nerve and sensory cervical rootlets. The area of numbness can reduce in size over time but can be permanent in some areas, especially the ear lobule. When level I is dissected, the marginal mandibular branch of the facial nerve is at risk. This branch can lie quite low in the neck as part of the investing fascia of the submandibular gland. Care to preserve this nerve branch even when defining the top of level II minimizes the risk, but weakness still results up to $23 \%$ of the time (37). Most weakness is temporary and results in an asymmetrical smile and short-term problems drinking from a glass until accommodation is made. Permanent marginal nerve weakness can be improved with facial plastic surgical procedures.

Uncommon injuries encountered during lateral neck dissection include: phrenic nerve injury, brachial plexus injury, cervical sympathetic trunk, and thoracic duct injury. The phrenic nerve lies on the anterior scalene muscle deep to the carotid sheath and should be identified and preserved. Similarly, the brachial plexus, which provides motor and sensory innervation to the upper extremity, enters the neck between the anterior and middle scalene muscles and is invested in the deep cervical fascia. It is usually unaffected by lateral neck dissection. Injury to the cervical sympathetic nerves can result in Horner's syndrome characterized by ipsilateral ptosis, miosis, and anhidrosis (38). Chyle fistula occurs when the thoracic duct in the left neck or the right neck cervical lymphatic duct is injured. Low level IV lymph nodes are typically involved in DTC and this area must be addressed to meticulously identify and avoid injuring or to ligate these lymphatic tributaries. These areas become especially problematic when bulky disease exists in low level IV or $\mathrm{Vb}$ requiring extensive dissection. Careful observation during a Valsalva maneuver or abdominal compression to elicit any leak in this area should be performed with ligation of this tissue when necessary. This complication can significantly increase utilization of inpatient resources and should be dealt with as best as possible at the time of neck dissection (39).

\section{Rationale for evaluation and treatment of the lateral neck in DTC}

Optimal management of patients with thyroid cancer requires removal of macroscopic clinical cervical lymph node metastases at the time of initial surgery and a comprehensive surveillance strategy to detect recurrent local and metastatic disease that may develop afterward. In accordance with ATA Thyroid cancer guidelines (14) recommendations 27a and 28, lateral neck dissection should be performed only as a therapeutic intervention for known disease. A prophylactic lateral neck dissection alone for thyroid cancer has not been proven effective to improve survival (40). There are significant risks to lateral neck dissection which have been outlined above, that render prophylactic lateral neck dissection unwarranted. The modalities and approach to establishing metastatic disease in the lateral neck follow.

Physical examination evaluation of the lateral neck for metastatic disease screens for visible or palpable lymph nodes. Medical records, including prior operative notes and pathology reports, and slides should be obtained for review in recurrent/persistent cancer cases. Imaging modalities, such as US (with or without FNAB) which allows for mapping of bilateral central and lateral neck compartments, iodine scans, $\mathrm{CT}$, hybrid imaging modalities such as single photon emission computed tomography/CT and positron emission tomography / $\mathrm{CT}$, technetium-99m methoxyisobutylisonitrile scintigraphy (MIBI scan), and magnetic resonance imaging can each be important in the assessment of the lateral neck. US performed by experienced hands is considered by most clinicians, and by the ATA, as the screening and surveillance imaging modality of choice for detection of lateral neck metastases (Table 2) (14) (ATA Thyroid cancer guidelines recommendations 21 and 22).

Timing of lateral neck dissection for well-DTC is less critical than is the central neck dissection for thyroid cancer or lateral neck dissection for squamous cell carcinoma. Proponents of routine prophylactic central neck dissection emphasize that an omission to perform it may result in a revision surgery being necessary that is more challenging due to entrance into the same operative field as the thyroidectomy with its resultant scar tissue (41). Negative neck US findings in DTC imply that the lateral neck be managed expectantly. As recommended by the ATA thyroid cancer guidelines (14), recommendation 48, surveillance imaging of the lateral neck should include ultrasonography. In cases when a lateral neck node is enlarged ( $>1.5 \mathrm{~cm}$ in levels I and IIa or $>1.0 \mathrm{~cm}$ in levels $\mathrm{Ilb}-\mathrm{Vb}$ ) or has sonographic features worrisome for disease, an US-guided fine-needle aspiration should be attempted to confirm disease including possibly testing the aspirate for $\mathrm{Tg}$ in a paucicellular specimen $(1,3,31)$. Those nodes that have suspicious US characteristics and are not amenable to FNAB may be observed for growth with serial US at intervals recommended by the ATA before planning intervention.

If the node is growing on follow-up US and is still inaccessible for biopsy, an open biopsy may be undertaken to establish a diagnosis. An open biopsy of a lymph node should only be performed as an excisional biopsy with frozen section analysis to help determine if a lateral neck dissection is necessary at that time. Excisional biopsy alone without a plan to do a formal lateral neck dissection at the same time, if the frozen section is positive, is less desirable due to the complexity involved in reoperative lateral neck dissection surgery.

According to the ATA thyroid cancer guidelines (14), recommendation 48 , nodes less than $5-8 \mathrm{~mm}$, especially without worrisome features, might be observed rather than attempting an FNAB. The attendant risk(s) of lateral neck dissection should always be weighed against the possible benefit.

Lateral neck dissection performed for macroscopic DTC metastases should be the selective neck dissection of levels IIa, III, IV, and Vb. "Berry picking" is not recommended. Axial CT or MR may be useful in cases of extensive nodal disease to be vigilant of nodes that might be present in the retropharynx or upper mediastinum. Metastatic thyroid disease to levels I is infrequent; therefore, dissection of level I is usually not indicated (42).

Dissection of level I puts the marginal mandibular branch of the facial nerve at risk, which might result in a weak lower lip. Dissection above the accessory nerve (IIb) is generally not necessary unless there are suspicious lymph nodes at level IIb 
or in the high jugular region (IIa); this will help to minimize postoperative morbidity associated with "shoulder syndrome," a condition of shoulder girdle weakness, stiffness, and chronic pain that can arise when CN XI function is impaired (43). Routine elective dissection of level Va may also be unnecessary when US evaluation shows no suspicious Va lesions. This further reduces the risk of injury to the accessory nerve. Lateral neck dissection is generally very well tolerated in most patients, may not require a drain and doesn't extend hospitalization for the majority of patients undergoing thyroid surgery.

Complications from all surgery for thyroid cancer, including lateral neck dissections, can be minimized when the operation is performed by high-volume thyroid surgeons $(11,12,44,45)$. Thus, the surgeon's skill level, especially in performing lateral neck dissections for DTC, should always be considered when managing a patient that may require a lateral neck dissection.

\section{Discussion}

\section{Proposals for future thyroid cancer} management guidelines

As a result of our literature review, we propose that future guidelines for the management of DTC consider the following as they relate to disease or the possibility of disease in lateral neck:

- Routine prophylactic lateral neck dissection for thyroid cancer has not been proven to improve survival. Thyroid cancer guidelines in the future may articulate this observation in the context of the comprehensive management of the thyroid cancer patient.

- Current guidelines recommend that nodes less than 5-8 mm, especially without worrisome features, might be observed rather than attempting an FNA biopsy. Guideline recommendations in the future may address the consideration of biopsy of any highly suspicious lymph node in the lateral neck without regard to size if a positive FNA would change clinical management.

- Unless findings are present to indicate dissection of compartments I or IIb, a routine dissection of these levels may not be necessary. Routine elective dissection of level Va may also be unnecessary when US evaluation shows no suspicious Va nodes, further reducing the risk of injury to the accessory nerve. Guidelines in the future may mention lateral neck levels requiring greatest attention during dissection.

- In cases of lateral neck recurrence, a comprehensive neck dissection of levels IIa, III, IV, and Vb should be performed. In revision lateral neck dissection, focus upon levels of demonstrable recurrence may be appropriate. Guideline recommendations in the future may delete previous radiation alone as a contraindication to comprehensive lateral neck dissection since external beam radiotherapy is a rare adjuvant used for DTC and comprehensive neck dissection after radiation is feasible and routinely done for other disease processes.

\section{Summary}

The lateral neck lymph nodes are a significant consideration in the surgical management of patients with DTC. Metastases to these nodes must be considered in the evaluation of the newly diagnosed thyroid cancer patient, and for surveillance of the previously treated DTC patient. Initial evaluation of the new DTC patient with US, or other modalities when indicated, will help to identify lateral neck lymph nodes of concern (46). Positive imaging findings should be addressed using US guided FNAB to confirm lateral neck disease and a comprehensive neck dissection of levels IIa, III, IV, and Vb should be performed when indicated.

\section{Acknowledgments}

The authors wish to acknowledge Dr. Jennifer Shinn and Ms. Susan Steelman for their advice in preparing and executing the systematic literature search strategy for lateral neck metastases from thyroid cancer; Dr. Doug Evans for helpful suggestions to the article; Dr. Gerard M. Doherty, Cochair of the ATA Surgical Affairs Committee at the time of the creation of this subcommittee; Dr. Robert Udelsman for financial support of the artistic rendering of Figure 1.

\section{Author Disclosure Statement}

No competing financial interests exist.

\section{References}

1. Kim E, Park JS, Son KR, Kim JH, Jeon SJ, Na DG 2008 Preoperative diagnosis of cervical metastatic lymph nodes in papillary thyroid carcinoma: comparison of ultrasound, computed tomography, and combined ultrasound with computed tomography. Thyroid 18:411-418.

2. Noguchi S, Noguchi A, Murakami N 1970 Papillary carcinoma of the thyroid. I. Developing pattern of metastasis. Cancer 26:1053-1060.

3. Stulak JM, Grant CS, Farley DR, Thompson GB, van Heerden JA, Hay ID, Reading CC, Charboneau JW 2006 Value of preoperative ultrasonography in the surgical management of initial and reoperative papillary thyroid cancer. Arch Surg 141:489-494; discussion 94-96.

4. Arturi F, Russo D, Giuffrida D, Ippolito A, Perrotti N, Vigneri R, Filetti S 1997 Early diagnosis by genetic analysis of differentiated thyroid cancer metastases in small lymph nodes. J Clin Endocrinol Metab 82:1638-1641.

5. Bardet S, Malville E, Rame JP, Babin E, Samama G, De Raucourt D, Michels JJ, Reznik Y, Henry-Amar M 2008 Macroscopic lymph-node involvement and neck dissection predict lymph-node recurrence in papillary thyroid carcinoma. Eur J Endocrinol 158:551-560.

6. Lundgren CI, Hall P, Dickman PW, Zedenius J 2006 Clinically significant prognostic factors for differentiated thyroid carcinoma: a population-based, nested case-control study. Cancer 106:524-531.

7. Podnos YD, Smith D, Wagman LD, Ellenhorn JD 2005 The implication of lymph node metastasis on survival in patients with well-differentiated thyroid cancer. Am Surg 71:731734 .

8. Leboulleux S, Rubino C, Baudin E, Caillou B, Hartl DM, Bidart JM, Travagli JP, Schlumberger M 2005 Prognostic factors for persistent or recurrent disease of papillary thyroid carcinoma with neck lymph node metastases and/or tumor extension beyond the thyroid capsule at initial diagnosis. J Clin Endocrinol Metab 90:5723-5729.

9. Mazzaferri EL, Young RL 1981 Papillary thyroid carcinoma: a 10 year follow-up report of the impact of therapy in 576 patients. Am J Med 70:511-518. 
10. Loh KC, Greenspan FS, Gee L, Miller TR, Yeo PP 1997 Pathological tumor-node-metastasis (pTNM) taging for papillary and follicular thyroid carcinomas: a retrospective analysis of 700 patients. J Clin Endocrinol Metab 82:35533562.

11. Grant CS, Stulak JM, Thompson GB, Richards ML, Reading CC, Hay ID 2010 Risks and adequacy of an optimized surgical approach to the primary surgical management of papillary thyroid carcinoma treated during 1999-2006. World J Surg 34:1239-1246.

12. Al-Saif O, Farrar WB, Bloomston M, Porter K, Ringel MD, Kloos RT 2010 Long-term efficacy of lymph node reoperation for persistent papillary thyroid cancer. J Clin Endocrinol Metab 95:2187-2194.

13. Carty SE, Cooper DS, Doherty GM, Duh QY, Kloos RT, Mandel SJ, Randolph GW, Stack BC, Jr., Steward DL, Terris DJ, Thompson GB, Tufano RP, Tuttle RM, Udelsman R 2009 Consensus statement on the terminology and classification of central neck dissection for thyroid cancer. Thyroid 19:1153-1158.

14. Cooper DS, Doherty GM, Haugen BR, Kloos RT, Lee SL, Mandel SJ, Mazzaferri EL, McIver B, Pacini F, Schlumberger M, Sherman SI, Steward DL, Tuttle RM 2009 Revised American Thyroid Association management guidelines for patients with thyroid nodules and differentiated thyroid cancer. Thyroid 19:1167-1214.

15. Machens A, Hauptmann S, Dralle H 2009 Lymph node dissection in the lateral neck for completion in central nodepositive papillary thyroid cancer. Surgery 145:176-181.

16. Roh JL, Kim JM, Park CI 2008 Lateral cervical lymph node metastases from papillary thyroid carcinoma: pattern of nodal metastases and optimal strategy for neck dissection. Ann Surg Oncol 15:1177-1182.

17. Shaha AR 2008 Complications of neck dissection for thyroid cancer. Ann Surg Oncol 15:397-399.

18. Roh JL, Kim DH, Park CI 2008 Prospective identification of chyle leakage in patients undergoing lateral neck dissection for metastatic thyroid cancer. Ann Surg Oncol 15:424-429.

19. Roh JL, Park JY, Park CI 2007 Total thyroidectomy plus neck dissection in differentiated papillary thyroid carcinoma patients: pattern of nodal metastasis, morbidity, recurrence, and postoperative levels of serum parathyroid hormone. Ann Surg 245:604-610.

20. Cheah WK, Arici C, Ituarte PH, Siperstein AE, Duh QY, Clark OH 2002 Complications of neck dissection for thyroid cancer. World J Surg 26:1013-1016.

21. Lee L, Steward DL 2008 Sonographically-directed neck dissection for recurrent thyroid carcinoma. Laryngoscope 118:991-994.

22. Gharib H, Papini E, Paschke R, Duick DS, Valcavi R, Hegedus L, Vitti P 2010 American Association of Clinical Endocrinologists, Associazione Medici Endocrinologi, and European Thyroid Association medical guidelines for clinical practice for the diagnosis and management of thyroid nodules: executive summary of recommendations. Endocr Prac 16:468-475.

23. Choi YJ, Yun JS, Kook SH, Jung EC, Park YL 2010 Clinical and imaging assessment of cervical lymph node metastasis in papillary thyroid carcinomas. World J Surg 34:1494-1499.

24. Xing M, Westra WH, Tufano RP, Cohen Y, Rosenbaum E, Rhoden KJ, Carson KA, Vasko V, Larin A, Tallini G, Tolaney S, Holt EH, Hui P, Umbricht CB, Basaria S, Ewertz M, Tufaro AP, Califano JA, Ringel MD, Zeiger MA, Sidransky D,
La, Ladenson PW 2005 BRAF mutation predicts a poorer clinical prognosis for papillary thyroid cancer. J Clin Endocrinol Metab 90:6373-6379.

25. Leboulleux S, Girard E, Rose M, Travagli JP, Sabbah N, Caillou B, Hartl DM, Lassau N, Baudin E, Schlumberger M 2007 Ultrasound criteria of malignancy for cervical lymph nodes in patients followed up for differentiated thyroid cancer. J Clin Endocrinol Metab 92:3590-3594.

26. Salmaslıoğlu A, Erbil Y, Citlak G, Ersöz F, Sarı S, Olmez A, Tunacı M,Yılmazbayhan D, Colak N, Ozarmağan S 2011 Diagnostic value of thyroglobulin measurement in fineneedle aspiration biopsy for detecting metastatic lymph nodes in patients with papillary thyroid carcinoma. Langenbecks Arch Surg 396:77-81.

27. Robbins KT, Medina JE, Wolfe GT, Levine PA, Sessions RB, Pruet CW 1991 Standardizing neck dissection terminology. Arch Otolaryngol Head Neck Surg 117:601-605.

28. Shah JP, Stiang E, Spiro RH, Vikiam B 1981 Neck dissection: current status and future possibilities. Clin Bull 11: 25-33.

29. Robbins KT, Clayman G, Levine PA, Medina J, Sessions R, Shaha A, Som P, Wolf GT; American Head and Neck Society; American Academy of Otolaryngology-Head and Neck Surgery 2002 Neck dissection classification update: revisions proposed by the American Head and Neck Society and the American Academy of Otolaryngology-Head and Neck Surgery. Arch Otolaryngol Head Neck Surg 128:751758.

30. Robbins KT, Shaha AR, Medina JE, Califano JA, Wolf GT, Ferlito A, Som PM, Day TA; Committee for Neck Dissection Classification, American Head and Neck Society 2008 Consensus statement on the classification and terminology of neck dissection. Arch Otolaryngol Head Neck Surg 134:536538.

31. Som PM, Curtin HD, Mancuso AA 2000 Imaging-based nodal classification for evaluation of neck metastatic adenopathy. AJR AM J Roentgenol 174:837-844.

32. Crile, G 1906 Excision of cancer of the head and neck with special reference to the plan of dissection in 132 operations. JAMA 47:1780-1786.

33. Crile G 1987 Landmark article Dec 1, 1906: excision of cancer of the head and neck. With special reference to the plan of dissection based on one hundred and thirty-two operations. JAMA 11:258:3286-3293.

34. Rubello D, Mazzarotto D, Carsaro D 2000 The role technetium-99m methoxyisobutylisonitrile scintigraphy in the planning of therapy and follow-up of patients with differentiated thyroid carcinoma after surgery. Eur J Nucl Med 27:431-440.

35. Rubello D, Salvatori M, Casar D, Piotto A, Toniato A, Gross MD, Al-Nahhas A, Muzzio PC, Pelizzo MR 2007 99m Tc-sestamibi radio-guided surgery of loco-regional 131Iodinenegative recurrent thyroid cancer. Eur J Surg Onc 33:902906.

36. Hillel AD, Kroll H, Dormon J, Medielos J 1989 Radical neck dissection: a subjective and objective evaluation of postoperative disability. J Otolaryngol 18:53-61.

37. Batstone MD, Scott B, Lowe D, Rogers SN 2009 Marginal mandibular nerve injury during neck dissection and its impact on patient perception of appearance. Head Neck 31:673-678.

38. Prim MP, De Diego JI, Verdaguer JM, Sastre N, Rabanal I 2006 Neurological complications following functional neck dissection. Eur Arch Otorhinolaryngol 263:473-476. 
39. Ilczyszyn A, Ridha H, Durrani AJ 2011 Management of chyle leak post neck dissection: A case report and literature review. J Plast Reconstr Aesthet Surg 64:e223-e230.

40. Moreno MA, Agarwal G, de Luna R, Siegel ER, Sherman SI, Edeiken-Monroe BS, Clayman GL 2011 Preoperative lateral neck ultrasonography as a long-term outcome predictor in papillary thyroid cancer. Arch Otolaryngol Head Neck Surg 137:157-162.

41. Kim MK, Mandel SH, Baloch Z, Livolsi VA, Langer JE, Didonato L, Fish S, Weber RS 2004 Morbidity following central compartment reoperation for recurrent or persistent thyroid cancer. Arch Otolaryngol Head Neck Surg 130:1214-1216.

42. Wu G, Fraser S, Pai SI, Farrag TY, Ladenson PW, Tufano RP 2011 Determining the extent of lateral neck dissection necessary to establish regional disease control and avoid reoperation after previous total thyroidectomy and radioactive iodine for papillary thyroid cancer. Head Neck 3 Nov 2011 [Epub ahead of print]; doi: 10.1002/hed.21937

43. Farrag T, Lin F, Brownlee N, Kim M, Sheth S, Tufano RP 2009 Is routine dissection of level II-B and V-A necessary in patients with papillary thyroid cancer undergoing lateral neck dissection for FNA-confirmed metastases in other levels. World J Surg 33:1680-1683.

44. Sosa JA, Bowman HM, Tielsch JM, Powe NR, Gordon TA, Udelsman R 1998 The importance of surgeon experience for clinical and economic outcomes from thyroidectomy. Ann Surg 228:320-330.

45. Stavrakis AI, Ituarte PH, Ko CY, Yeh MW 2007 Surgeon volume as a predictor of outcomes in inpatient and outpatient endocrine surgery. Surgery 142:887-899; discussion 99.

46. Jeong HS, Baek CH, Son YI, Choi JY, Kim HJ, Ko YH, Chung JH, Baek HJ 2006 Integrated 18F-FDG PET/CT for the initial evaluation of cervical node level of patients with papillary thyroid carcinoma: comparison with ultrasound and contrast-enhanced CT. Clin Endocrinol 65:402-407.

47. Kaplan SL, Mandel SJ, Muller R, Baloch ZW, Thaler ER, Loevner LA 2009 The role of MR imaging in detecting nodal disease in thyroidectomy patients with rising thyroglobulin levels. AJNR Am J Neuroradiol 30:608-612.

48. Kupferman ME, Weinstock YE, Santillan AA, Mishra A, Roberts D, Clayman GL, Weber RS 2008 Predictors of level V metastasis in well-differentiated thyroid cancer. Head Neck 30:1469-1474.

49. Turanli S 2007 Is the type of dissection in lateral neck metastasis for differentiated thyroid carcinoma important? Otolaryngol Head Neck Surg 136:957-960.

50. Asai $Y$, Mineta $H$, Umemura $K$, Yasuhara A, Mukoudaka $H$, Ishizaki H, Ito H, Nozue M 1993 Statistical analysis on the cases of thyroid papillary carcinoma. Nippon Jibiinkoka Gakkai Kaiho 96:58-65.

51. Ardito G, Rulli F, Revelli L, Moschella F, Galata G, Giustozzi E, Ardito F, Farinon A 2005 A less invasive, selective, functional neck dissection for papillary thyroid carcinoma. Langenbecks Arch Surg 390:381-384.

52. Ito $Y$, Fukushima M, Tomoda $C$, Inoue H, Kihara M, Higashiyama T, Uruno T, Takamura Y, Miya 2A, Kobayashi K, Matsuzuka F, Miyauchi A 2009 Prognosis of patients with papillary thyroid carcinoma having clinically apparent metastasis to the lateral compartment. Endocr J 56:759-766.

53. Ito $Y$, Miyauchi A, Jikuzono T, Higashiyama T, Takamura $Y$, Miya A, Kobayashi K, Matsuzuka F, Ichihara K, Kuma K 2007 Risk factors contributing to a poor prognosis of papillary thyroid carcinoma: validity of UICC/AJCC TNM classification and stage grouping. World J Surg 31:838-848.

54. Baek SK, Jung KY, Kang SM, Kwon SY, Woo JS, Cho SH, Chung EJ 2010 Clinical risk factors associated with cervical lymph node recurrence in papillary thyroid carcinoma. Thyroid 20:147-152.

55. Chung YS, Kim JY, Bae JS, Song BJ, Kim JS, Jeon HM, Jeong SS, Kim EK, Park WC 2009 Lateral lymph node metastasis in papillary thyroid carcinoma: results of therapeutic lymph node dissection. Thyroid 19:241-246.

56. Kim JM, Kim TY, Kim WB, Gong G, Kim SC, Hong SJ, Shong YK 2006 Lymphovascular invasion is associated with lateral cervical lymph node metastasis in papillary thyroid carcinoma. Laryngoscope 116:2081-2085.

57. Alfalah H, Cranshaw I, Jany T, Arnalsteen L, Leteurtre E, Cardot C, Pattou F, Carnaille B 2008 Risk factors for lateral cervical lymph node involvement in follicular thyroid carcinoma. World J Surg 32:2623-2626.

58. Witte J, Goretzki PE, Dieken J, Simon D, Roher HD 2002 Importance of lymph node metastases in follicular thyroid cancer. World J Surg 26:1017-1022.

59. Guerrero MA, Suh I, Vriens MR, Shen WT, Gosnell J, Kebebew E, Duh QY, Clark OH 2010 Age and tumor size predicts lymph node involvement in Hürthle Cell Carcinoma. J Cancer 1:23-26.

60. Mills SC, Haq M, Smellie WJ, Harmer C 2009 Hürthle cell carcinoma of the thyroid: retrospective review of 62 patients treated at the Royal Marsden Hospital between 1946 and 2003. Eur J Surg Oncol 35:230-234.

61. Jung CK, Kang YG, Bae JS, Lim DJ, Choi YJ, Lee KY 2010 Unique patterns of tumor growth related with the risk of lymph node metastasis in papillary thyroid carcinoma. Mod Pathol 23:1201-1208.

62. Min HS, Choe G, Kim SW, Park YJ, Park DJ, Youn YK, Park SH, Cho BY, Park SY 2008 S100A4 expression is associated with lymph node metastasis in papillary microcarcinoma of the thyroid. Mod Pathol 21:748-755.

63. Siironen P, Louhimo J, Nordling S, Ristimaki A, Maenpaa H, Haapiainen R, Haglund C 2005 Prognostic factors in papillary thyroid cancer: an evaluation of 601 consecutive patients. Tumour Biol 26:57-64.

Address correspondence to: Brendan C. Stack, Jr., M.D., FACS, FACE Department of Otolaryngology/Head and Neck Surgery University of Arkansas for Medical Sciences 4301 W. Markham St., \#543 Little Rock, AR 72205

E-mail: bstack@uams.edu

Members of the American Thyroid Association Surgical Affairs Committee, Subcommittee for Lateral Neck Dissection for Well Differentiated Thyroid Cancer are (alphabetically listed) Brendan C. Stack, Jr., M.D. (Chair); Robert L. Ferris, M.D., Ph.D.; David Goldenberg, M.D.; Megan Haymart, M.D.; Ashok Shaha, M.D.; Sheila Sheth, M.D.; Julie Ann Sosa, M.D.; and Ralph P. Tufano, M.D. Dr. Brendan C. Stack, Jr., is the Chair of the Subcommittee and Dr. Ralph P. Tufano was the Cochair of the Surgical Affairs Committee. 